\title{
Application of signal processing techniques for islanding detection of distributed generation in distribution network: A review
}

\author{
Safdar Raza ${ }^{\text {a }}$, Hazlie Mokhlis ${ }^{\mathrm{a}, *}$, Hamzah Arof $^{\mathrm{a}}$, J.A. Laghari ${ }^{\mathrm{a}}$, Li Wang ${ }^{\mathrm{b}}$ \\ a Department of Electrical Engineering, Faculty of Engineering, University of Malaya, 50603 Kuala Lumpur, Malaysia \\ ${ }^{\mathrm{b}}$ Department of Electrical Engineering, College of Electrical Engineering \& Computer Science, National Cheng Kung University, Tainan City 70101, Taiwan
}

\section{A R T I C L E I N F O}

\section{Article history:}

Received 5 December 2014

Accepted 6 March 2015

Available online 23 March 2015

\section{Keywords:}

Islanding detection

Signal processing

Fourier transform

Wavelet transform

s-transform

Hilbert Huang transform

tt-transform

\begin{abstract}
A B S T R A C T
High penetration of distributed generation resources (DGR) in distribution network provides many benefits in terms of high power quality, efficiency, and low carbon emissions in power system. However, efficient islanding detection and immediate disconnection of DGR is critical in order to avoid equipment damage, grid protection interference, and personnel safety hazards. Islanding detection techniques are mainly classified into remote, passive, active, and hybrid techniques. From these, passive techniques are more advantageous due to lower power quality degradation, lower cost, and widespread usage by power utilities. However, the main limitations of these techniques are that they possess a large non detection zones and require threshold setting. Various signal processing techniques and intelligent classifiers have been used to overcome the limitations of passive islanding. Signal processing techniques, in particular, are adopted due to their versatility, stability, cost effectiveness, and ease of modification. This paper presents a comprehensive overview of signal processing techniques used to improve common passive islanding detection techniques. A performance comparison between the signal processing based islanding detection techniques with existing techniques are also provided. Finally, this paper outlines the relative advantages and limitations of the signal processing techniques in order to provide basic guidelines for researchers and field engineers in determining the best method for their system.
\end{abstract}

(c) 2015 Elsevier Ltd. All rights reserved.

\section{Introduction}

Due to the rapid increase of oil and natural gas prices, greenhouse effect, and other environmental issues, the installation of distributed generation resources (DGRs) has shed a new light on the field of electric power supply. It attracts industrial and commercial customers by providing a competitive environment with respect to new integrating technologies, environmental benefits, and reduced losses $[1,2]$. The DGRs, such as mini/micro hydro, wind turbines, photovoltaic and fuel cell increases the efficiency and stability of the distribution network [3]. However, the steady state and dynamic behavior of distributed energy resources causes technical challenges in control and protection [4]. The occurrence of islanding is one of the major issues in this context. In islanding condition, the part of the distribution system to which DGR is connected is electrically isolated from the utility grid but energized by the distributed generation resource [5]. Fig. 1 shows the islanding

* Corresponding author. Tel.: +60 379675238 (office), mobile: +60 1 26467936; fax: +600379675316 .

E-mail address: hazlie@um.edu.my (H. Mokhlis). phenomenon of a distribution network connected with three distribution generators (DGs).

If the utility supply is cut off, then an island is formed, as shown by dotted circles. Islanding creates many problems, such as complication in orderly power restoration, degradation of power quality, and endangering the lives of utility personnel. Due to these severe consequences, existing standards such as IEC 62116, IEEE 1547, IEEE 929-2000 and AS4777.3-2005 standards do not allow distributed generation resources to operate in islanded mode [6]. According to IEEE 1547 standard, the islanding condition should be detected and disconnected within $2 \mathrm{~s}$ from the distribution network [7].

According to aforementioned standards, the distributed generation resources are disconnected from the power system when islanding occurs. However, it is not an economical solution, because power generation from DGRs may fulfil the load demand, if not the whole demand, as part of its operation. In this regard, researchers are working expansively in order to make the islanding operation of distribution network a viable solution by properly addressing various technical issues [8].

The most important and the foremost step for successful islanding are to detect the islanding condition when the distribution 


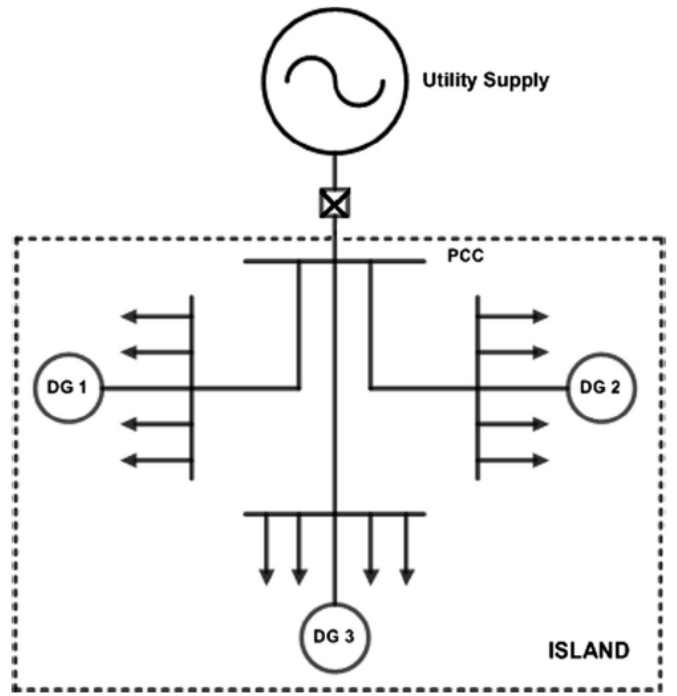

Fig. 1. Phenomenon of islanding occurrence in distribution network connected with three DG.

network is disconnected from the grid. It requires an efficient islanding detection technique in order to detect and to isolate the distributed energy resource. If the islanding condition is not detected accurately and timely, the whole distribution network might collapse. Thus, accuracy and time are the two most important factors in islanding detection. Several techniques have been proposed for this, and they are categorized as remote and local (passive, active, and hybrid) methods. However, each method is replete with its own merits and demerits [9]. Hence, the researchers shift their interests and intentions towards signal processing based islanding detection techniques, because these techniques have the ability to extract the unseen/hidden features of the measured signal that are helpful for islanding detection. The objective of this paper is to review the existing signal processing based islanding detection techniques.

The rest of the paper is organized in the following order. Section 2 elaborates the islanding detection techniques. Signal processing based islanding detection techniques, with and without intelligent classifiers, is covered under Sections 3 and 4. Some miscellaneous techniques and discussion is presented in Sections 5 and 6. Finally, the conclusion is presented in Section 7.

\section{Categorization of islanding detection methods}

Islanding detection techniques are mainly categorized into remote, local, and signal processing based, as shown in Fig 2 [10-12]. These methods are further classified into different techniques on the basis of different parameters, such as detection speed, error detection rate, power quality, non detection zone (NDZ), and efficacy in multiple inverter cases [13]. Comprehensive discussions of these techniques are presented in the following sections.

\subsection{Remote islanding detection techniques}

Remote islanding detection techniques works on the principle of communication between utility and distributed energy resource.
Once islanding occurs, a trip signal is sent to the distributed generation resource. Transfer trip scheme $[14,15]$ and Power line carrier communication (PLCC) scheme [16,17] falls under this category. These techniques have zero NDZ, faster response time, zero impact on power quality and system transients, high reliability, and works effectively in multiple DG systems. However, remote techniques are very expensive for implementation on small-scale systems [18]. Hence, local techniques are preferred for the aforementioned purpose.

\subsection{Local islanding detection techniques}

As the name indicates, it is based on measuring the variations in system parameters, such as frequency, voltage, impedance, phase angle, active power, reactive power, and harmonic distortion at the DG site for islanding detection. These techniques are further categorized into passive, active, and hybrid techniques. The number of proposed active and passive techniques increases rapidly over the last few years [9].

\subsection{Passive islanding detection techniques}

Passive islanding detection techniques basically monitor the system parameters, such as frequency, voltage, and harmonics at the point of common coupling, or at the DG terminals, and compare it with a predetermined threshold value for islanding detection. Fig. 3 shows the basic working principle of passive islanding detection techniques.

Some of the most common passive techniques are the rate of change of power (ROCOP) [7], rate of change of frequency (ROCOF) [19-21], rate of change of frequency over power (ROCOFOP) [22], change of impedance [23], voltage unbalance [24], over/under $(\mathrm{O} / \mathrm{U})$ voltage and over/under frequency [25], harmonic distortion (voltage and current) $[24,25]$, phase jump detection [26], rate of change of voltage and change in power factor [27]. The characteristics of these techniques are summarized in Table 1.

Cost effectiveness, fast detection speed, and no impact on power quality are some of the major advantages of passive techniques. However, a major problem with this technique is that it suffers from large non detection zone (NDZ), and it is very difficult to detect islanding when the generation and load in the islanded system are closely matched. Furthermore, the setting of threshold value requires special consideration. Lower threshold settings may result in nuisance tripping, and if the setting is too high, islanding may not be detected. Hence, error detection rates are high. These drawbacks can be overcome by using signal processing techniques and intelligent classifiers. However, these problems can also be overcome by active techniques [13,28].

\subsubsection{Active islanding detection techniques}

Active islanding detection techniques interact with the power system by introducing perturbations into the system variables, such as frequency, voltage, currents, and harmonics. Fig 4 shows the basic working principle of active islanding detection techniques. The impact of these perturbations is significant if the distributed generation resource is islanded otherwise quite negligible.

Some of the most common active techniques are reactive power export error detection (RPEED) [29], impedance measurement $[7,23]$, slip mode frequency shift method (SMS) [30,31], Active frequency drift (AFD) [32], frequency jump (FJ) [13], Active frequency drift with positive feedback (AFDPF) [33], sandia frequency shift (SFS) [34,35], sandia voltage shift (SVS) [36], variation of active and reactive power $[11,13,32]$, negative sequence current injection $[37,38]$, high frequency signal injection [39,40], virtual capacitor [41], virtual inductor [42] and phase PLL perturbation method 


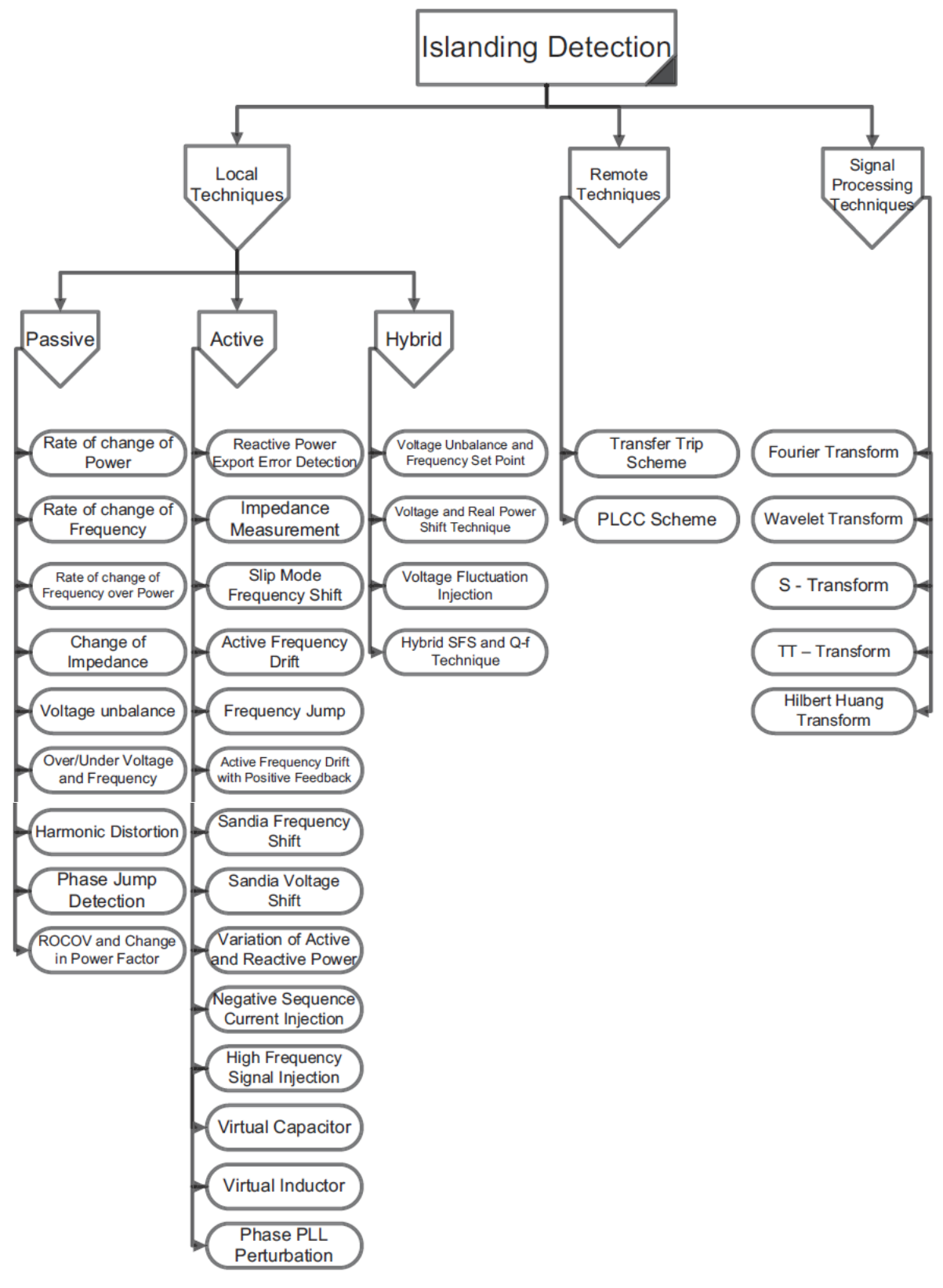

Fig. 2. Categorization of islanding detection techniques.

[43]. Most of these techniques are used for inverter type DGs. The characteristics of these techniques are summarized in Table 2.

In comparison to passive techniques, active techniques reduce the non-detection zone (NDZ) and decrease the error detection rate. Furthermore, in order to inject perturbations in the power systems, additional controllers/power electronics equipment is required. This increases the complexity of the system and reduces the power quality. Moreover, additional detection time is required in order to observe the power system response on perturbations. Hence, the system's stability is significantly degraded. 


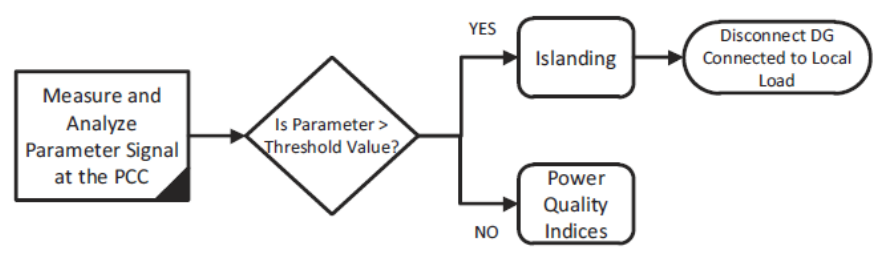

Fig. 3. Basic working principle of passive islanding detection techniques.

Table 1

Characteristics of different passive techniques.

\begin{tabular}{|c|c|c|c|c|}
\hline Method & $\begin{array}{l}\text { Detection } \\
\text { time }\end{array}$ & $\begin{array}{l}\text { Error } \\
\text { detection } \\
\text { rate }\end{array}$ & $\begin{array}{l}\text { Impact on } \\
\text { power quality }\end{array}$ & NDZ \\
\hline ROCOP & $24-26 \mathrm{~ms}$ & High & No & Small \\
\hline ROCOF & $24 \mathrm{~ms}$ & High & No & Small \\
\hline ROCOFOP & $100 \mathrm{~ms}$ & Low & No & $\begin{array}{l}\text { Smaller } \\
\text { than ROCOF }\end{array}$ \\
\hline $\begin{array}{l}\text { Change of } \\
\text { impedance }\end{array}$ & $10 \mathrm{~ms}$ & Low & No & Small \\
\hline $\begin{array}{l}\text { Voltage } \\
\text { unbalance }\end{array}$ & $53 \mathrm{~ms}$ & Low & No & Large \\
\hline $\begin{array}{l}\mathrm{O} / \mathrm{U} \text { voltage and } \\
\text { frequency }\end{array}$ & $\begin{array}{l}4 \mathrm{~ms} \text { to } \\
2 \mathrm{~s}\end{array}$ & Low & No & Large \\
\hline $\begin{array}{l}\text { Harmonic } \\
\text { distortion }\end{array}$ & $45 \mathrm{~ms}$ & High & No & $\begin{array}{l}\text { Large for } \\
\text { high } Q\end{array}$ \\
\hline Phase jump & $10-20 \mathrm{~ms}$ & Low & No & Large \\
\hline
\end{tabular}

2.3.2. Hybrid islanding detection techniques

Hybrid islanding detection techniques basically works on the combined features of the aforementioned techniques and is effectively applied to complex systems. During islanding detection, the passive technique functions as primary, while the active technique functions as a secondary, as shown in Fig 5. Hence, the combination of these methods will improve the multiple performance indices.

Some examples of hybrid islanding detection techniques include voltage unbalance and frequency set point method [44], technique based on voltage and real power shift [45], voltage fluctuation injection [46], hybrid SFS and Q-f technique [47], and the technique based on rate of change of reactive power and load connecting strategy [48].

These techniques possess very small non-detection zone (NDZ), and degradation in power quality is also reduced because perturbations are only introduced when islanding is suspected. However, this combination increases the cost of the system, along with the islanding detection time.

From the above discussion, it is noticed that each islanding detection technique has its own merits and demerits. However, the issues that are still unresolved include accuracy, very high detection speed, and compatibility of detecting islanding in multiple and hybrid DGs environment. In this regard, it can be noted from the aforementioned review that passive islanding detection techniques have the potential to fulfil these requirements, provided that their limitation are solved using some other means. Passive islanding detection techniques have the advantage of not degrading the power quality, capable of detecting islanding quickly, and are compatible for all types of DGs. However, their main limitation is the large non detection zone and threshold settings. Both of these limitations can be addressed by using signal processing techniques and intelligent classifiers.

\section{Signal processing techniques for islanding detection}

Signal processing techniques are commonly used to improve the performance of passive islanding detection techniques. Versatility, stability, cost effectiveness, and ease of modification properties of the signal processing techniques help researchers extract the hidden characteristics of the measured signals for islanding detection. On the basis of these extracted features, decision can be made on whether islanding did occur. Fig. 6 shows the basic steps involved in islanding detection using signal processing techniques.

The basic signal processing tools, which are used for islanding detections, are Fourier transform, s-transform, Hilbert Huang transform, wavelet transform, and tt-transform. The description of these signal processing tools used in islanding detection techniques are discussed in the following sections.

\subsection{Fourier transform based islanding detection techniques}

Fourier transform is the most common technique in frequency domain analysis. It basically represents a signal as a summation of sinusoidal terms of different frequencies. It extracts the features of the stationary signal at specific frequencies, but is incapable of detecting the time distribution of different frequencies. It is also unable to resolve any momentary information associated with fluctuations [49]. Hence, the time-frequency analysis is proposed. The short time Fourier transform (STFT) is the modification of Fourier transform. It divides the signal into small frames, where each frame can be assumed to be stationary. These numerous frames of the signal are evaluated by the moving window. This moving window identifies the relation between the time and the change in frequency [50]. However, STFT cannot analyze the nonstationary signal due to the limitation of fixed window's width [51].

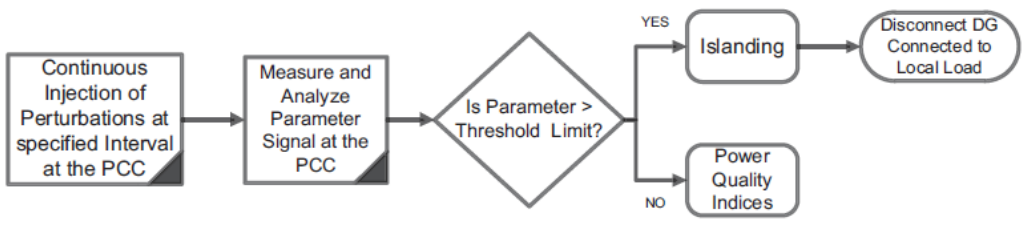

Fig. 4. Basic working principle of active islanding detection techniques. 
Table 2

Characteristics of different active techniques.

\begin{tabular}{|c|c|c|c|c|}
\hline Techniques & $\begin{array}{l}\text { Detection } \\
\text { time }\end{array}$ & $\begin{array}{l}\text { Error } \\
\text { detection } \\
\text { rate }\end{array}$ & $\begin{array}{l}\text { Impact on } \\
\text { power } \\
\text { quality }\end{array}$ & NDZ \\
\hline RPEED & $2 \mathrm{~s}$ & Low & Degrades & Small \\
\hline $\begin{array}{l}\text { Impedance } \\
\text { measurement }\end{array}$ & $\begin{array}{l}0.77- \\
0.95 \mathrm{~s}\end{array}$ & Low & Degrades & Small \\
\hline SMS & $\begin{array}{l}0.4 \mathrm{~s} \\
\text { (approx) }\end{array}$ & Low & Degrades & Small \\
\hline AFD & With $2 \mathrm{~s}$ & High & Degrades & $\begin{array}{l}\text { Large if } \\
\text { value of } Q \text { is } \\
\text { high }\end{array}$ \\
\hline FJ & $75 \mathrm{~ms}$ & Low & Degrades & Small \\
\hline AFDPF & $\begin{array}{l}1 \mathrm{~s} \\
\text { (approx) }\end{array}$ & $\begin{array}{l}\text { Lower } \\
\text { than AFD }\end{array}$ & $\begin{array}{l}\text { Slightly } \\
\text { degrades }\end{array}$ & $\begin{array}{l}\text { Smaller than } \\
\text { AFD }\end{array}$ \\
\hline SFS & $0.5 \mathrm{~s}$ & Low & $\begin{array}{l}\text { Slightly } \\
\text { degrades }\end{array}$ & Smallest \\
\hline SVS & $0.5 \mathrm{~s}$ & Low & $\begin{array}{l}\text { Slightly } \\
\text { degrades }\end{array}$ & Smallest \\
\hline $\begin{array}{l}\text { Variation of active } \\
\text { and reactive } \\
\text { power }\end{array}$ & $0.3-0.75 \mathrm{~s}$ & High & Degrades & Small \\
\hline $\begin{array}{l}\text { Negative sequence } \\
\text { current injection }\end{array}$ & $60 \mathrm{~ms}$ & Low & Degrades & None \\
\hline $\begin{array}{l}\text { High frequency } \\
\text { signal injection }\end{array}$ & Few ms & Low & $\begin{array}{l}\text { Slightly } \\
\text { degrades }\end{array}$ & Smallest \\
\hline Virtual capacitor & $20-51 \mathrm{~ms}$ & Low & $\begin{array}{l}\text { Slightly } \\
\text { degrades }\end{array}$ & Smallest \\
\hline Virtual inductor & $13-59 \mathrm{~ms}$ & Low & $\begin{array}{l}\text { Slightly } \\
\text { degrades }\end{array}$ & Smallest \\
\hline $\begin{array}{l}\text { Phase PL } \\
\text { perturbation }\end{array}$ & $120 \mathrm{~ms}$ & Low & Negligible & Smallest \\
\hline
\end{tabular}

Discrete Fourier transform (DFT) is very dominant technique for frequency domain analysis of discrete time signals. It transforms the discrete time sequence of finite length into discrete frequency sequence of finite length. Fast Fourier transform (FFT) also gives similar results as the DFT, although in lesser amounts of time. However, it is not suitable for the analysis of non-stationary signals, due to the fact that it depicts those spectral values that do not exist in the original signal. Kim [52] introduces a new passive technique for islanding detection based on DFT for the extraction of the desired features. Conventional passive techniques misinterpret the grid disturbances, such as sag, swell, and transient conditions. NDZ might also have occurred if the power generated by photovoltaic (PV) and the load matches. The proposed method uses the variations in 2nd harmonic components of grid voltage. The utilization of harmonic coefficients provides robust control against grid disturbances and also reduces the NDZ. The islanding detection time is around $1 \mathrm{~ms}$ via the usage of high performance DSP controller.

In order to overcome the problem of slow/reduced computation time of DFT, Goertzel algorithm is used. It is basically a type of discrete Fourier transform and the fastest technique of pitch detection compared to FFT and DFT. It works from the perspective of filtering operation at a specified frequency, or from the perspective of the
DFT taken over short time section of the signal. It directly calculates the amplitude and phase of the desired frequency of the input signal, which ultimately reduces the computational time [53-55]. Jae-Hung et al. [56] uses Goertzel algorithm to reduce the islanding detection time in single phase 2 stage Photovoltaic (PV) system. In the proposed system, the inverter injects the output current with a ninth harmonic component into the grid, and detects the same in voltage at the point of common coupling. NDZ does not exist in this method, even under perfect match of power and load. The impact on the power quality is also negligible, and islanding is detected within 2 cycles.

\subsection{Wavelet transform based islanding detection techniques}

The wavelet transform (WT) is also used for the analysis of the signal. It is a mathematical model based on square integral and group theory, similar to FT. It decomposes a signal into its constituents at different frequency scales $[57,58]$. It represents a signal in both time and frequency domains. Hence, it is suitable for examining the signals in those applications where time-frequency resolution is considered necessary. It has been widely used in power system applications, such as detection, disturbance transition events in electrical power quality, feature extraction, power system protection, and de-noising [59-61].

The wavelet transform is more advantageous compared to Fourier based transforms (STFT, FFT and DFT).This is due to the fact that window size is fixed in Fourier based transform, but varies in wavelet transform. Hence, time-frequency resolutions are not compromised. Furthermore, wavelet transform determines the time and frequency information simultaneously for low and high frequencies by long and short windows, respectively [62]. Wavelet transform is categorised into continuous (CWT) and discrete wavelet transforms (DWT). CWT is used in islanding detection by analysing DG voltage. The Mallat decomposition is also used to extract and eliminate the noise from the signal [63]. This method reduces the computational efficiency by introducing the numerous coefficients. This problem diverts the attention of the researchers towards DWT.

In $[64,65]$, authors used DWT to analyze the voltage signal from a single phase PV system. The proposed method uses Bi-orthogonal 1.5 and 5 decomposition levels for islanding detection. The reduction in the number of sensors, minimisation in computational burden and complexity are some of the advantages associated with this technique. Daubechies mother wavelet based DWT is used in [66]. It examines the variations in voltage and frequency. The salient features of this method are the simplicity in programming, enhancement of islanding detection capability, and simultaneous observation of power quality profiles. The proposed scheme is tested and verified in several scenarios with flexibility, feasibility, and robustness. In [67], negative sequence of current and voltage signals are considered by Daubechies db4 based DWT. The standard deviation and change in energy coefficients discriminates between islanding and other disturbance conditions. In the

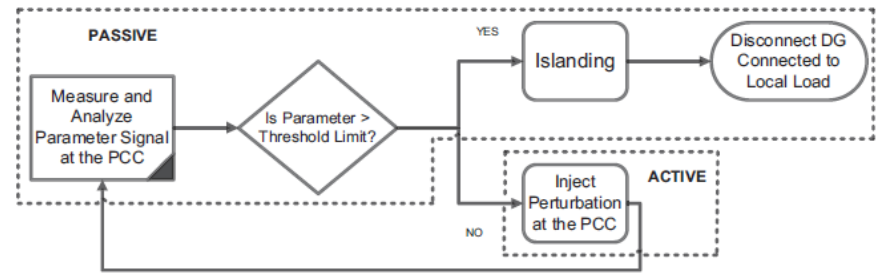

Fig. 5. Basic working principle of hybrid techniques. 


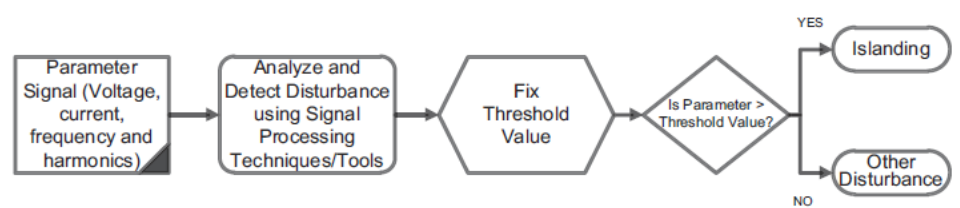

Fig. 6. Block diagram for signal processing based islanding detection technique.

proposed method, islanding is detected in 1 cycle by using the first level of energy and standard deviations. Researchers benefit from the compactness and localisation properties of the Daubechies $\mathrm{db} 4$ in diminishing NDZ. The proposed scheme is compared with the existing passive (over/under voltage and frequency) scheme, and found very efficient and effective in all working condition [68]. Karegar et al. [69] proposes the DWT for the islanding detection of wind turbines. Voltage profiles are examined by $\mathrm{db} 5$. The proposed scheme proves dependability under different load conditions. In [70], db4 DWT-based technique is utilised for grid connected PV DGs. The spectral changes in the higher frequency components of voltage are analyzed for islanding detection. The proposed scheme is found very effective, and detected islanding conditions within $0.05 \mathrm{~s}$. Sharma et al. [71] utilises the localisation and compactness property of the Dyadic wavelet transform for islanding detection. It has been found that the proposed scheme discriminates the islanding and non-islanding scenarios for grid connected PV system in more than 1 cycle. Liu et al. [72] introduces the islanding detection scheme based on WT for the stand alone operation of DG system.

The main disadvantages associated with DWT are the integration of the high frequencies and measurement of several electrical quantities. Therefore, wavelet packet transform (WPT) is proposed in [73]. This scheme proposes a new index called node rate of change of power index. This index computes the change of the power at each WPT sub band. The base of WPT is $\mathrm{db} 10$, because it has a smaller number of wavelet coefficients. In [74], the "Haar" mother wavelet has been used for islanding detection. This type of mother wavelet requires the least decomposition levels, thus, has the least detection time. This method calculates the current signal at the point of common coupling (PCC) and detects the islanding within $5.5 \mathrm{~ms}$. The proposed method is also applicable for multi-DG environment.

In [75], a new feature extraction technique is proposed for islanding detection. This work examine the variations in harmonic profiles for inverter based DG system, and is basically an extension of [65]. Wavelet transform-based multi-resolution analysis (WTbased MRA) technique is used for feature extraction. The WT-based MRA decomposes the output voltage into multiple scales. Each scale produces a sequence of wavelet coefficients (WCs) on the bases of frequency bandwidth. The change in the ratio of the WCs is used for islanding detection. The simulation results prove that the proposed scheme is successful for islanding detection under all operating conditions.

Samui and Samantaray in [76,77] proposes a new technique for islanding detection based on wavelet singular entropy (WSE). WSE integrates the advantages of wavelet transform, singular value decomposition, and Shanon entropy. In the proposed scheme, the wavelet transform analyzes the three phase voltage signal and generates the detailed coefficients. Singular value matrix is calculated from these detailed coefficients in order to determine the WSE for each phase. Finally, WSE index is produced by adding all the phases of WSE. The proposed scheme is compared with the two existing schemes (rate of change of frequency (ROCOF) and rate of change of power (ROCOP)) in a perfectly matching environment, and it has been found that the proposed scheme detects islanding condition effectively within $10 \mathrm{~ms}$.

\section{3. s-transform based islanding detection techniques}

Wavelet transform extracts the desired features of the signal from both time and frequency domains. However, batch processing and noise sensitivity are the disadvantages associated with this technique. To overcome these problems, Stockwell proposes stransform in 1996. In this technique, the properties of both STFT and WT are merged. It is a time-frequency technique having a variable window of STFT and an expansion of WT. It is based on a scalable localising Gaussian window, and supplies the frequency dependent resolution [78-80]. It provides multi-resolution, and keeps the phase of each frequency component unchanged. It transforms the signal from the time domain to two dimensional frequency domains. Local spectral characteristics are examined either by the amplitude time frequency spectrum, or by the phase time frequency spectrum. In this tool, the sinusoid is fixed with respect to the time axis, and localised scalable Gaussian window examined it more comprehensively by dilating and translating the phase frequency $[81,82]$. s-transform (ST) provides considerable and noteworthy results in the detection and localisation of disturbances due to islanding or any other condition. Thus, it detects the disturbance signal efficiently and accurately.

In [83], the islanding detection technique using s-transform is proposed for the hybrid system. It clearly shows the domination of s-transform in comparison to wavelet transform for islanding detection on the bases of the simulation results. Ray et al. [84,85] extracts the negative sequence voltage for islanding detection. They also compared the wavelet transform and s-transform under noisy conditions. On the basis of the simulation results, it has been found that s-transform determine the islanding scenario proficiently, within 26-28 ms. Islanding detection, on the basis of performance indices, was also conducted, which is another contribution to this field. A new technique that relies on s-transform based cumulative sum detector (CUSUM) is proposed in [86]. In this proposed technique, the spectral energy contents of the negative sequence voltage and current signals are used for computation. It has been verified that the proposed technique detects the islanding condition in $25 \mathrm{~ms}$ with an accuracy of more than $92 \%$.

The problem associated with s-transform is that its performance weakens under certain operating situations, such as transients. The s-transform is modified to hyperbolic s-transform to overcome the adverse effect of transients and to realize a superior signal processing technique $[87,88]$. Compared to s-transform, the hyperbolic s-transform has a pseudo Gaussian hyperbolic window. It has frequency dependence in its shape in integration to its width and height. This asymmetrical window provides better resolution in both time and frequency at high and low frequencies, respectively. In [89], hyperbolic s-transform is used to detect the islanding condition. The change in energy and standard deviation (STD) of the perturbed voltage signal at PCC is resolute, and on the basis of these predicated values; a suitable threshold is finalised in order

\section{Link to Full-Text Articles :}

\section{http://www.sciencedirect.com/science/article/pii/S0196890415002393}

https://www.infona.pl/resource/bwmeta1.element.elsevier-c2b8de16-f3a5-3792-8291-c80db07764bf 